\title{
Neutron powder diffraction study of $\mathrm{NaMn}_{2} \mathrm{O}_{4}$ and $\mathrm{Li}_{0.92} \mathrm{Mn}_{2} \mathrm{O}_{4}$ : Insights on spin-charge-orbital ordering
}

\author{
N. Matsubara $\odot,{ }^{1,}{ }^{*}$ E. Nocerino, ${ }^{1}$ K. Kamazawa, ${ }^{2}$ O. K. Forslund $\odot,{ }^{1}$ Y. Sassa $\odot,{ }^{3}$ L. Keller $\odot,{ }^{4}$ V. V. Sikolenko $\odot,{ }^{5,6}$ \\ V. Pomjakushin, ${ }^{4}$ H. Sakurai, ${ }^{7}$ J. Sugiyama ${ }^{\circ},{ }^{2}$ and M. Månsson ${ }^{1}{ }^{1, \dagger}$ \\ ${ }^{1}$ Department of Applied Physics, KTH Royal Institute of Technology, 10691 Stockholm, Sweden \\ ${ }^{2}$ Neutron Science and Technology Center, Comprehensive Research Organization for Science and Society (CROSS), \\ Tokai, Ibaraki 319-1106, Japan \\ ${ }^{3}$ Department of Physics, Chalmers University of Technology, 41296 Gothenburg, Sweden \\ ${ }^{4}$ Laboratory for Neutron Scattering and Imaging, Paul Scherrer Institut, 5232 Villigen PSI, Switzerland \\ ${ }^{5}$ Joint Institute for Nuclear Research, 141980 Dubna, Russia \\ ${ }^{6}$ Karlsruhe Institute of Technology, 76131 Karlsruhe Germany \\ ${ }^{7}$ National Institute for Materials Science, Namiki 1-1, Tsukuba, Ibaraki 305-0044, Japan
}

(Received 29 June 2020; accepted 28 September 2020; published 28 October 2020)

\begin{abstract}
High-pressure synthesized quasi-one-dimensional $\mathrm{NaMn}_{2} \mathrm{O}_{4}$ and $\mathrm{Li}_{0.92} \mathrm{Mn}_{2} \mathrm{O}_{4}$ are both antiferromagnetic insulators. Here their atomic and magnetic structures are investigated using neutron powder diffraction. The present crystal structural analyses of $\mathrm{NaMn}_{2} \mathrm{O}_{4}$ reveal that a $\mathrm{Mn}^{3+} / \mathrm{Mn}^{4+}$ charge-ordering state exists even at low temperature (down to $1.5 \mathrm{~K}$ ). It is evident that one of the Mn sites shows a strongly distorted $\mathrm{Mn}^{3+}$ octahedron due to the Jahn-Teller effect. Above $T_{N}=35 \mathrm{~K}$, a two-dimensional short-range correlation is observed, as indicated by asymmetric diffuse scattering. Below $T_{N}$, two antiferromagnetic transitions are observed: (i) a commensurate long-range $\mathrm{Mn}^{3+}$ spin ordering below $T_{N 1}=35 \mathrm{~K}$ and (ii) an incommensurate $\mathrm{Mn}^{4+}$ spin ordering below $T_{N 2}=11 \mathrm{~K}$. Surprisingly, the two antiferromagnetic orders are found to be independent of each other. The commensurate magnetic structure $\left(k_{\mathrm{C}}=0.5,0.5,0.5\right)$ follows the magnetic anisotropy of the local easy axes of $\mathrm{Mn}^{3+}$, while the incommensurate $\mathrm{Mn}^{4+}$ one shows a spin-density-wave or a cycloidal order with $k_{\mathrm{IC}}=(0,0,0.216)$. For $\mathrm{Li}_{0.92} \mathrm{Mn}_{2} \mathrm{O}_{4}$, on the other hand, the absence of a long-range spin-ordered state is confirmed down to $1.5 \mathrm{~K}$.
\end{abstract}

DOI: 10.1103/PhysRevResearch.2.043143

\section{INTRODUCTION}

Low-dimensional and frustrated spin systems have attracted attention in experimental and theoretical research fields $[1,2]$. In particular, the compounds with a calciumferrite $\mathrm{CaFe}_{2} \mathrm{O}_{4}$ structure (CFO), so-called postspinels, have been extensively studied in the past decade because of the complexity of their competing interactions in a magnetic arrangement [3-8]. The crystal structure of CFO is based on edge-sharing $\mathrm{Fe}$ octahedron chains running along the $c$ axis and are connected with each other by corner sharing in the $a b$ plane. These six chains build up a distorted hexagonal void (tunnels), where $\mathrm{Ca}$ ions are situated (see Fig. 3). They pose a geometrically frustrated lattice, based on a honeycomblike mesh of triangular or zigzag ladders with magnetic ions. Such a frustrated system is known to show intriguing magnetic ground states and physical properties, e.g., spin glasses,

\footnotetext{
*namim@kth.se
}

†condmat@kth.se

Published by the American Physical Society under the terms of the Creative Commons Attribution 4.0 International license. Further distribution of this work must maintain attribution to the author(s) and the published article's title, journal citation, and DOI. Funded by Bibsam. spin liquids, and multiferroicity $[9,10]$. In fact, the related CFO-NaCr $2 \mathrm{O}_{4}$ exhibit an unconventional colossal magnetoresistance effect below the Néel temperature $\left(T_{N} \sim 125 \mathrm{~K}\right)$ $[11,12]$. Here a combined study using positive muon spin rotation and relaxation $\left(\mu^{+} \mathrm{SR}\right)$ and neutron powder diffraction (NPD) has clarified the presence of two-dimensional (2D) antiferromagnetic (AFM) coupling among 1D zigzag $\mathrm{Cr}_{2} \mathrm{O}_{4}$ chains $[13,14]$.

Furthermore, a tunnel structure along the $c$ axis in the CFO lattice provides a $1 \mathrm{D}$ conduction pathway for cations [see the structure in Fig. 3(a)]. Hence, the CFO compounds with $\mathrm{Li}^{+}$ and $\mathrm{Na}^{+}$ions are expected to show high ionic conductivity. This makes the CFO compounds attractive also for use as solid-state electrolyte material in future all-solid-state batteries $[6,15-19]$.

When $\mathrm{Li}^{+}$and $\mathrm{Na}^{+}$occupy the $A$ site in a CFO structure represented by $A B_{2} \mathrm{O}_{4}(A=\mathrm{Li}$ and $\mathrm{Na}$ and $B=\mathrm{Mn}, \mathrm{Cr}$, and $\mathrm{V})$, the remaining $B$ ions should be in a mixed valence state with $B^{3+}$ and $B^{4+}$. According to the previous work on CFO- $\mathrm{NaV}_{2} \mathrm{O}_{4}$ [20], $\mathrm{NaCr}_{2} \mathrm{O}_{4}$ [11], and $\mathrm{Li}_{0.92} \mathrm{Mn}_{2} \mathrm{O}_{4}$ [15,21], there is no indication for charge ordering in the zigzag chains. Instead, trivalent and tetravalent cations occupy the octahedral $B$ site randomly to keep local charge neutrality. However, at room temperature $\mathrm{NaMn}_{2} \mathrm{O}_{4}$ exhibits the $\mathrm{Mn}^{3+} / \mathrm{Mn}^{4+}$ charge ordering, evidenced by the undistorted $\mathrm{Mn}^{+4} \mathrm{O}_{6}$ along with 

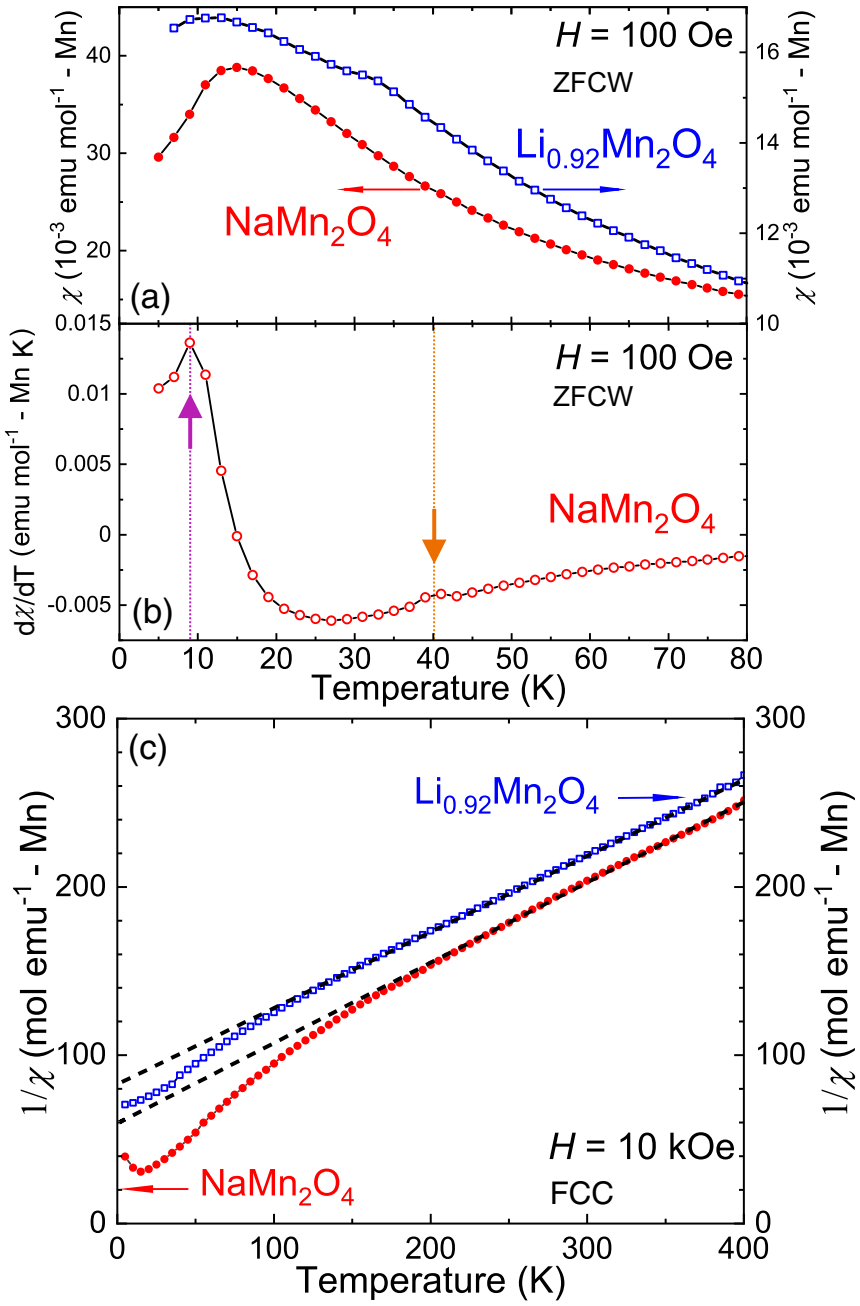

FIG. 1. (a) Magnetic susceptibility curves $\chi(T)$ of $\mathrm{NaMn}_{2} \mathrm{O}_{4}$ (red closed circles, left axis) and $\mathrm{Li}_{0.92} \mathrm{Mn}_{2} \mathrm{O}_{4}$ (blue squares, right axis) recorded using a zero-field-cooled warming ( $\mathrm{ZFCW}$ ) protocol under an external magnetic field of 100 Oe. (b) Corresponding differential susceptibility $(d \chi / d T)(T)$ curve of $\mathrm{NaMn}_{2} \mathrm{O}_{4}$, indicating two magnetic transitions. (c) Plot of $1 / \chi(T)$ with Curie-Weiss fitting (dotted lines) recorded using a field-cooled cooling (FCC) protocol under an external magnetic field of $10 \mathrm{kOe}$.

strongly distorted $\mathrm{Mn}^{+3} \mathrm{O}_{6}$ due to the Jahn-Teller (JT) effect $[8,22]$.

The magnetic properties of both $\mathrm{NaMn}_{2} \mathrm{O}_{4}$ and $\mathrm{Li}_{0.92} \mathrm{Mn}_{2} \mathrm{O}_{4}$ were previously studied using bulk magnetization and $\mu^{+} \mathrm{SR}$ measurements [23]. The magnetization vs temperature $[M(T)]$ curve for the current $\mathrm{NaMn}_{2} \mathrm{O}_{4}$ sample batch [Fig. 1(a)] exhibits a clear maximum at $15 \mathrm{~K}$, indicating the presence of an AFM transition, while the $M(T)$ curve for $\mathrm{Li}_{0.92} \mathrm{Mn}_{2} \mathrm{O}_{4}$ shows a weak anomaly below $40 \mathrm{~K}$. As can be seen in Fig. 1(c), the inverse susceptibility curves show a large linear domain, for which Curie-Weiss fitting leads to $\mu_{\text {eff }}$ and $\Theta_{\mathrm{p}}$ values $\sim 3.35 \mu_{\mathrm{B}} / \mathrm{Mn}$ and $\sim-49.6 \mathrm{~K}$ for $\mathrm{NaMn}_{2} \mathrm{O}_{4}$ and $\sim 4.00 \mu_{\mathrm{B}} / \mathrm{Mn}$ and $\sim-161 \mathrm{~K}$ for $\mathrm{Li}_{0.92} \mathrm{Mn}_{2} \mathrm{O}_{4}$ [23].

The $\mu^{+} \mathrm{SR}$ measurements under a weak transverse field reveal that $T_{N}=39 \mathrm{~K}$ for $\mathrm{NaMn}_{2} \mathrm{O}_{4}$ and $44 \mathrm{~K}$ for $\mathrm{Li}_{0.92} \mathrm{Mn}_{2} \mathrm{O}_{4}$. However, the zero-field $\mu^{+}$SR spectrum lacks a clear oscillation caused by the formation of a static internal magnetic field for both $\mathrm{NaMn}_{2} \mathrm{O}_{4}$ and $\mathrm{Li}_{0.92} \mathrm{Mn}_{2} \mathrm{O}_{4}$. This suggests that the internal AFM field is too rapidly fluctuating and the muons are unable to see it, i.e., it is out of the range of the $\mu^{+} \mathrm{SR}$ time window. Therefore, in order to further study the AFM nature in $\mathrm{NaMn}_{2} \mathrm{O}_{4}$ and $\mathrm{Li}_{0.92} \mathrm{Mn}_{2} \mathrm{O}_{4}$, the neutron diffraction technique is necessary, particularly for clarifying the difference between $\mathrm{NaMn}_{2} \mathrm{O}_{4}$ and $\mathrm{Li}_{0.92} \mathrm{Mn}_{2} \mathrm{O}_{4}$.

In this article, we report a systematic NPD study of both $\mathrm{NaMn}_{2} \mathrm{O}_{4}$ and $\mathrm{Li}_{0.92} \mathrm{Mn}_{2} \mathrm{O}_{4}$. For $\mathrm{NaMn}_{2} \mathrm{O}_{4}$, the crystal structure down to $1.5 \mathrm{~K}$ is confirmed as a charge and Jahn-Teller orbital ordered state, as reported previously by room-temperature single-crystal $\mathrm{x}$-ray-diffraction measurements [8]. Above the magnetic transition temperature, diffuse scattering corresponding to a $2 \mathrm{D}$ short-range spin ordering is observed between 40 and $75 \mathrm{~K}$. Below $40 \mathrm{~K}$, the magnetic Bragg peaks, which are indexed by using a commensurate propagation vector $k_{\mathrm{C}}=(0.5,0.5,0.5)$, start to grow with decreasing temperature. Below $11 \mathrm{~K}$, another set of magnetic Bragg peaks appears at incommensurate positions indexed with $k_{\mathrm{IC}}=(0,0,0.216)$. Based on the NPD data, we propose two AFM sublattices to explain the AFM ground state in $\mathrm{NaMn}_{2} \mathrm{O}_{4}$. As for no-charge ordered $\mathrm{Li}_{0.92} \mathrm{Mn}_{2} \mathrm{O}_{4}$, our neutron diffraction results reveal an absence of long-range magnetic ordering down to $1.5 \mathrm{~K}$.

\section{EXPERIMENTAL METHODS}

\section{A. Synthesis}

$\mathrm{NaMn}_{2} \mathrm{O}_{4}$ was prepared by a solid-state reaction under high pressure, starting from $\mathrm{Na}_{2} \mathrm{O}_{2}$ and $\mathrm{Mn}_{2} \mathrm{O}_{3}$ powders [8]. A mixture of the two powders was packed in an Au capsule in an Ar-filled glove box, before being heated at $1300^{\circ} \mathrm{C}$ for $1 \mathrm{~h}$ under $P=6 \mathrm{GPa}$, finally quenched to room temperature. For CFO-type $\mathrm{Li}_{0.92} \mathrm{Mn}_{2} \mathrm{O}_{4}$, a spinel-type $\mathrm{Li}_{0.92} \mathrm{Mn}_{2} \mathrm{O}_{4}$ was at first prepared by a conventional solid-state reaction using $\mathrm{Li}_{2} \mathrm{CO}_{3}$ and $\mathrm{Mn}_{2} \mathrm{O}_{3}$ powders [21]. A mixture of precursors was heated at $850^{\circ} \mathrm{C}$ at ambient pressure. After, the obtained spinel-type $\mathrm{Li}_{0.92} \mathrm{Mn}_{2} \mathrm{O}_{4}$ powder was packed in an Au capsule, heated at $1300^{\circ} \mathrm{C}$ for $1 \mathrm{~h}$ under $P=6 \mathrm{GPa}$, and finally quenched to room temperature.

\section{B. Magnetic susceptibility}

Magnetization measurements as a function of temperature were performed with a superconducting quantum interference device magnetometer (MPMS, Quantum Design) in zerofield-cooled warming or field-cooled warming or field-cooled cooling modes in the temperature range between 5 and $300 \mathrm{~K}$.

\section{Neutron powder diffraction}

Neutron powder diffraction patterns were recorded on the HRPT [24] and DMC diffractometers [Paul Scherrer Institute (PSI), Switzerland] with wavelengths $\lambda=1.886$ and $1.494 \AA$ and $\lambda=2.45$ and $4.20 \AA$, respectively, in the temperature range between 6 and $200 \mathrm{~K}$. For both experiments the very same sample batches were investigated and the samples were sealed inside 6-mm-diam vanadium sample cans. Data analysis and Rietveld refinements were performed with software tools from the FULLPROF SUITE [25]. Symmetry analysis was 


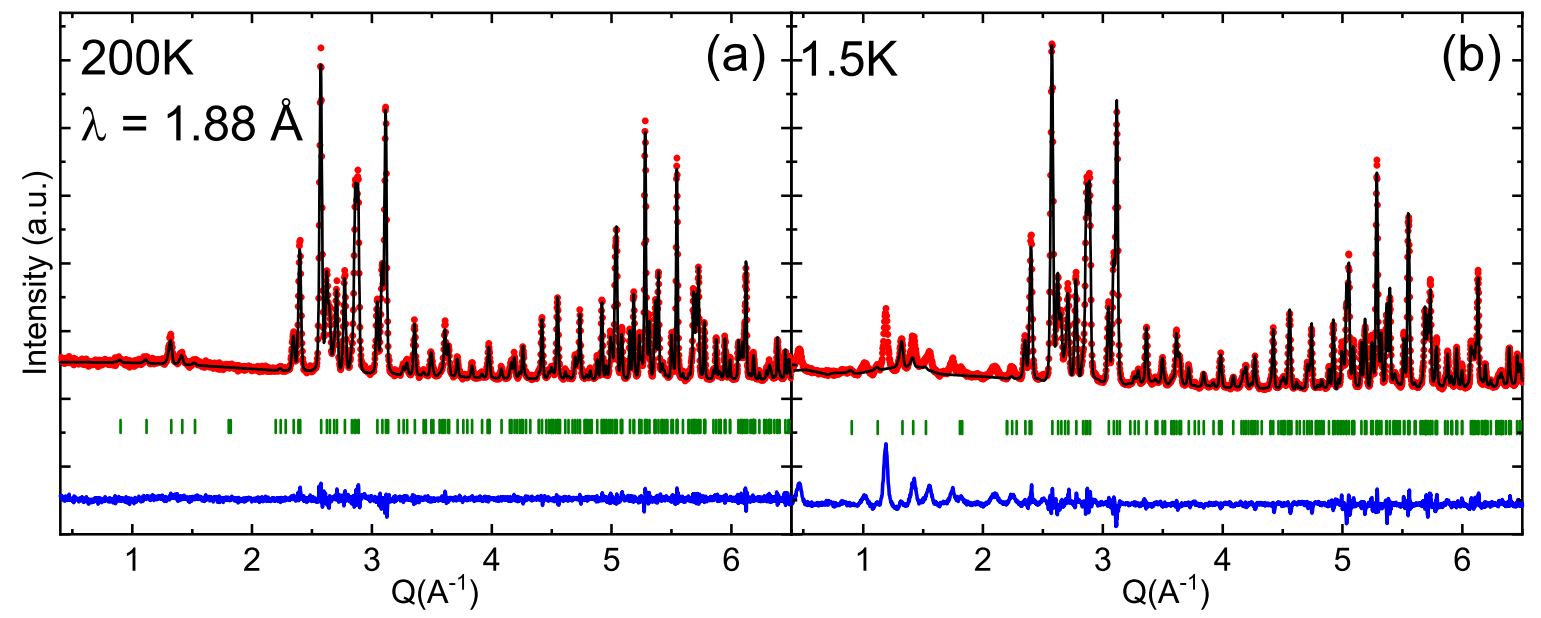

FIG. 2. Rietveld refinement of neutron diffraction data recorded on HRPT (PSI) of $\mathrm{NaMn}_{2} \mathrm{O}_{4}$ at (a) $T=200 \mathrm{~K}$ and (b) $T=1.5 \mathrm{~K}$ using $\lambda=1.88 \AA$. Note that the magnetic peaks present in the $1.5 \mathrm{~K}$ diffraction pattern are here left unindexed for the structural refinement. Experimental data are shown by open red circles, the calculated profile is shown by a solid black line, and allowed Bragg reflections are shown by vertical green marks. The difference between the experimental and calculated profiles is displayed at the bottom of the graph (solid blue line), where a series of magnetic peaks is clearly visible.

carried out using the Bilbao Crystallographic Server [26,27] and ISODISTORT [28].

\section{RESULTS}

\section{A. Crystal structure of $\mathrm{NaMn}_{2} \mathrm{O}_{4}$}

The crystal structure of $\mathrm{NaMn}_{2} \mathrm{O}_{4}$ at $200 \mathrm{~K}$ was refined in the orthorhombic Pnam space group (No. 62) with $a=$ 8.870(1) $\AA, b=11.227(1) \AA$, and $c=2.843(1) \AA$, starting from the reported unit cell and atomic coordinates [8]. This structural model provides excellent fitting to the highresolution NPD data recorded at HRPT (Fig. 2 and Table I), and the corresponding crystal structure is illustrated in Fig. 3. This confirms that $\mathrm{NaMn}_{2} \mathrm{O}_{4}$ is in a CFO-type structure with a charge ordering of $\mathrm{Mn}^{3+}\left(t_{2 g}^{3} e_{g}^{1}\right)$ and $\mathrm{Mn}^{4+}\left(t_{2 g}^{3} e_{g}^{0}\right)$. In the CFO structure, two different $\mathrm{Mn}_{2} \mathrm{O}_{4}$ zigzag chains are formed by a network of edge-sharing $\mathrm{MnO}_{6}$ octahedra (Mn-Mn bond labeled as $J_{2}$ ) aligned along the $c$ axis [Figs. 3(a) and 3(c)]. These four zigzag chains are linked by corner-sharing octahedra to form a 3D framework structure and make an irregular hexagonal 1D tunnel along the $c$ axis, in which $\mathrm{Na}^{+}$ions are located. Depending on the direction, this connects equivalent Mn sites via next nearest-neighbor Mn1 $\left(J_{1}\right)$ and $\operatorname{Mn} 2\left(J_{3}\right)$, along with nonequivalent $\mathrm{Mn}$ sites through $J_{4}$ and $J_{5}$ (see Fig. 3).

The $\mathrm{Mn}^{3+}$ and $\mathrm{Mn}^{4+}$ ions are fully ordered (within the refinement standard deviation), in contrast to the other CFOtype $\mathrm{Na}^{3+} B^{4+} \mathrm{O}_{4}(B=\mathrm{V}, \mathrm{Ti}$, and $\mathrm{Cr})$ materials. As a results, in the $\mathrm{NaMn}_{2} \mathrm{O}_{4}$ lattice, two crystallographically different $\mathrm{Mn}$ sites are clearly distinguishable, which are coordinated by six oxygen atoms [Fig. 3(b)]. As already reported in [8], the $\mathrm{Mn}^{3+} \mathrm{O}_{6}$ octahedron (manganese labeled as Mn1) is strongly (a)

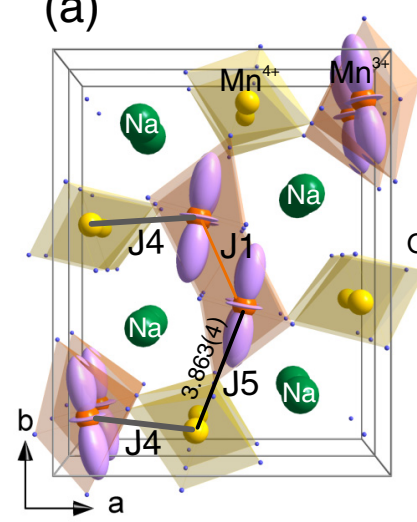

(b)

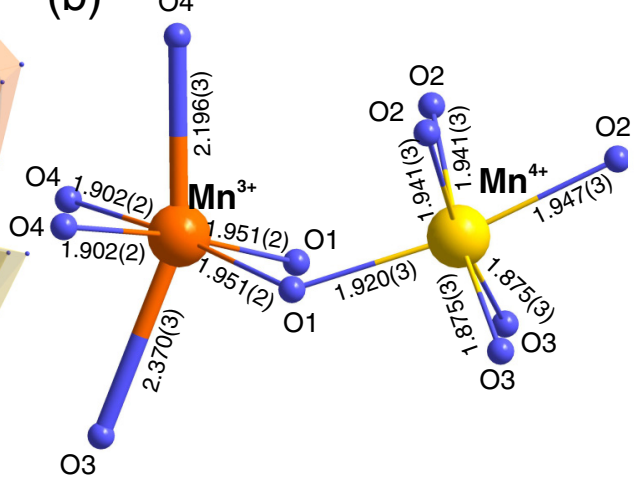

(c)

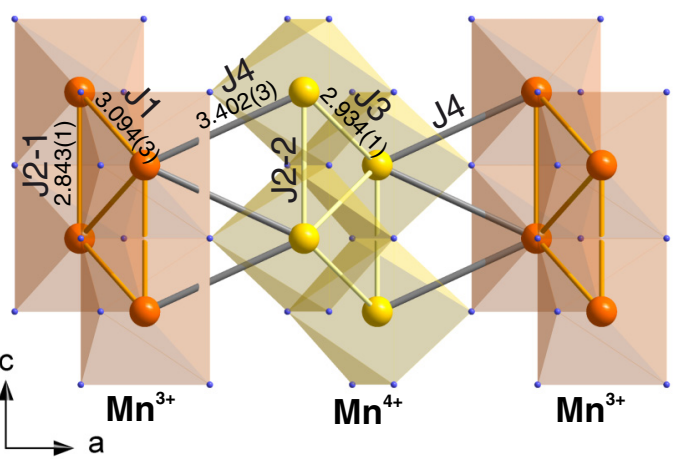

FIG. 3. Polyhedron representation of the CFO-type $\mathrm{NaMn}_{2} \mathrm{O}_{4}$ structure projected (a-b) along [001] and (c) [010]. The structure shows a charge ordered state of $\mathrm{Mn}^{3+} / \mathrm{Mn}^{4+}$ (orange and yellow polyhedra/atoms, respectively), Na (green atoms), and the $d_{z^{2}}$ orbital ordering (purple ellipsoid). (b) Bond distances (in angstroms) from structural refinements of NPD data at $T=200 \mathrm{~K}$. Thick lines highlight the elongated $\mathrm{Mn}^{3+}-\mathrm{O}$ distances. In (c), for clarity, only a part of a chain is shown. The possible different magnetic exchanges paths, i.e., Mn-Mn bonds $\left(J_{\mathrm{i}}\right)$, are shown by lines. The thick gray and black bonds show the connections between $\mathrm{Mn}^{3+}$ and $\mathrm{Mn}^{4+}$ ladders corresponding to $J_{4}$ and $J_{5}$, respectively. The orange and yellow bonds indicate links between the same Mn sites. 
TABLE I. Structural parameters of $\mathrm{NaMn}_{2} \mathrm{O}_{4}$ at 200 and $1.5 \mathrm{~K}$ from Rietveld refinements of HRPT data. The space group is Pnam and all the atoms are at site $4 c(x, y, 1 / 4)$.

\begin{tabular}{|c|c|c|c|}
\hline Site & Parameter & $200 \mathrm{~K}$ & $1.5 \mathrm{~K}$ \\
\hline & $a(\AA)$ & $8.870(1)$ & $8.861(1)$ \\
\hline & $b(\AA)$ & $11.227(1)$ & $11.197(1)$ \\
\hline & $c(\AA)$ & $2.843(1)$ & $2.841(1)$ \\
\hline & $V\left(\AA^{3}\right)$ & 283.1 (1) & 281.9 (1) \\
\hline \multirow[t]{3}{*}{$\mathrm{Na}$} & $x$ & $0.2473(4)$ & $0.2472(4)$ \\
\hline & $y$ & $0.3345(3)$ & $0.3352(3)$ \\
\hline & $B\left(\AA^{2}\right)$ & $0.52(6)$ & $0.33(6)$ \\
\hline \multirow[t]{3}{*}{ Mn1 } & $x$ & $0.0714(3)$ & $0.0716(4)$ \\
\hline & $y$ & $0.1086(3)$ & 0.1088 \\
\hline & $B\left(\AA^{2}\right)$ & $0.01(6)$ & $0.01(6)$ \\
\hline \multirow[t]{3}{*}{ Mn2 } & $x$ & $0.0806(4)$ & 0.0807 (4) \\
\hline & $y$ & $0.5949(3)$ & $0.5956(3)$ \\
\hline & $B\left(\AA^{2}\right)$ & $0.01(6)$ & $0.14(7)$ \\
\hline \multirow[t]{3}{*}{$\mathrm{O} 1$} & $x$ & $0.2864(2)$ & $0.2870(2)$ \\
\hline & $y$ & $0.6478(2)$ & $0.6478(2)$ \\
\hline & $B\left(\AA^{2}\right)$ & 0.09 (4) & 0.08 (4) \\
\hline \multirow[t]{3}{*}{$\mathrm{O} 2$} & $x$ & $0.3832(2)$ & $0.3849(3)$ \\
\hline & $y$ & 0.9809 (2) & $0.9802(2)$ \\
\hline & $B\left(\AA^{2}\right)$ & $0.09(4)$ & $0.21(4)$ \\
\hline \multirow[t]{3}{*}{$\mathrm{O} 3$} & $x$ & $0.4747(2)$ & $0.4745(2)$ \\
\hline & $y$ & $0.1947(2)$ & $0.1942(2)$ \\
\hline & $B\left(\AA^{2}\right)$ & $0.30(4)$ & 0.35 (4) \\
\hline \multirow[t]{5}{*}{$\mathrm{O} 4$} & $x$ & $0.0683(2)$ & 0.0689 \\
\hline & $y$ & $0.9132(2)$ & $0.9135(2)$ \\
\hline & $B\left(\AA^{2}\right)$ & $0.16(4)$ & $0.29(4)$ \\
\hline & $R_{\text {Bragg }}(\%)$ & 4.19 & 4.69 \\
\hline & $\chi_{\text {fit }}^{2}$ & 2.17 & 5.34 \\
\hline
\end{tabular}

elongated due to stabilization of the $d_{z^{2}}$ orbital, i.e., JahnTeller distortion of the $\mathrm{Mn}^{3+}$ ions. That is, among the six Mn1-O bonds of the $\mathrm{Mn}^{3+} \mathrm{O}_{6}$ octahedron, four Mn1-O distances ranges between approximately 1.90 and $1.95 \AA$ and two Mn1-O distances are larger, spanning from approximately 2.19 to $2.37 \AA$, resulting in an average distance of $2.05 \AA$ at $200 \mathrm{~K}$, as summarized in Table II. The stabilized $d_{z^{2}}$ orbitals are aligned in the $a b$ plane and form a zigzag pattern on the [001] projection, consisting of alternating ladder rows with two different orbital orientations [Fig. 3(a)]. Within the ladders, the distorted triangular lattice is formed by the $\mathrm{Mn}^{3+}$ ions as illustrated in Fig. 3(c). More-detailed three-edges of each triangular lattice correspond to one short Mn1-Mn1 distance of $2.84 \AA\left(J_{2-1}\right)$ and two longer Mn1-Mn1 distances of $3.09 \AA\left(J_{1}\right)$. In this edge-sharing octahedron network, the halffilled $t_{2 \mathrm{~g}}$ orbitals are directed along the short Mn-Mn distance in the edge-sharing octahedra $\left(J_{2-1}\right)$. This should clearly favor strong antiferromagnetic direct exchange interaction over a superexchange interaction through the Mn-O-Mn pathway (with an angle of $\sim 90^{\circ}$ ) [29].

In contrast, the $\mathrm{Mn}^{4+} \mathrm{O}_{6}$ octahedron (labeled as Mn2) only possesses a very small distortion with the average distance between $\mathrm{Mn} 2$ and $\mathrm{O}$ of $1.92 \AA$. In order to compare the distortion of an octahedron quantitatively, one could use the
TABLE II. Selected distances (in angstroms) and angles (in degrees) in $\mathrm{NaMn}_{2} \mathrm{O}_{4}$ at 200 and $1.5 \mathrm{~K}$. The distortion parameter is calculated for each manganese octahedron as $\Delta d=$ $1 / 6 \sum_{n=1,6}\left[\left(d_{n}-\langle d\rangle\right) /\langle d\rangle\right]^{2}$, with $\langle d\rangle$ being the average distance between Mn and O. The bond valence sum (BVS) is also indicated.

\begin{tabular}{lcc}
\hline \hline Selected distance $(\AA)$ and angles $\left({ }^{\circ}\right)$ & $200 \mathrm{~K}$ & $1.5 \mathrm{~K}$ \\
\hline Mn1-O1 & $1.951(2)$ & $1.950(4)$ \\
Mn1-O3 & $2.370(3)$ & $2.372(6)$ \\
Mn1-O4 & $2.196(3)$ & $2.188(6)$ \\
Mn1-O4 & $1.902(2)$ & $1.909(4)$ \\
average & $2.046(1)$ & $2.046(2)$ \\
$\Delta d \times 10^{-4}$ & 74.4 & 72.6 \\
BVS & $3.05(1)$ & $3.04(2)$ \\
Mn2-O1 & $1.920(3)$ & $1.911(6)$ \\
Mn2-O2 & $1.947(3)$ & $1.952(6)$ \\
Mn2-O2 & $1.941(3)$ & $1.956(4)$ \\
Mn2-O3 & $1.875(2)$ & $1.853(4)$ \\
average & $1.916(1)$ & $1.914(2)$ \\
$\Delta d \times 10^{-4}$ & 2.537 & 5.723 \\
BVS & $3.87(1)$ & $3.92(2)$ \\
Mn-Mn along $c\left(J_{2-1}, J_{2-2}\right)$ & $2.843(1)$ & $2.841(1)$ \\
Mn1-Mn1 $\left(J_{1}\right)$ & $3.094(3)$ & $3.088(6)$ \\
Mn1-O1-Mn1 & $93.54(9)$ & $93.48(17)$ \\
Mn1-O4-Mn1 & $96.70(9)$ & $96.17(18)$ \\
Mn2-Mn2 $\left(J_{3}\right)$ & $2.934(4)$ & $2.957(6)$ \\
Mn2-O2-Mn2 & $94.19(11)$ & $93.12(16)$ \\
Mn2-O3-Mn2 & $98.62(10)$ & $100.12(16)$ \\
Mn1-Mn2 $\left(J_{4}\right)$ & $3.402(3)$ & $3.402(7)$ \\
Mn1-Mn2 $\left(J_{5}\right)$ & $3.863(4)$ & $3.833(6)$ \\
Mn1-O1-Mn2 & $123.0(2)$ & $123.5(4)$ \\
Mn1-O3-Mn2 & $130.7(2)$ & $129.9(4)$ \\
\hline \hline
\end{tabular}

distortion parameter

$$
\Delta d=1 / 6 \sum_{n=1,6}\left[\left(d_{n}-\langle d\rangle\right) /\langle d\rangle\right]^{2},
$$

which corresponds to the deviation of $\mathrm{Mn}-\mathrm{O}$ distances with respect to the average distance. In fact, the $\Delta d$ of $\mathrm{Mn}^{3+} \mathrm{O}_{6}$ is $74 \times 10^{-4}$, whereas for $\mathrm{Mn}^{4+} \mathrm{O}_{6}$, the $\Delta d$ is $2.5 \times 10^{-4}$, i.e., $\frac{1}{30}$. Note that the $\Delta d$ for many Jahn-Teller distorted manganites shows $\Delta d>30 \times 10^{-4}$ [30,31]. The triangular ladder of $\mathrm{Mn}^{4+}$ is also much more regular, as shown in Fig. 3(b) (Mn2-Mn2 distances 2.8-2.9 ̊), than that of $\mathrm{Mn}^{3+}$ (Mn1-Mn1 distances 2.8-3.1 ̊). The bond valence sum calculations for the manganese using the Zachariasen formula $\left[V_{i}=\sum_{j} \exp \left(d_{0}-d_{i j}\right) / 0.37\right]$ [32] are also in fair agreement with expected values (see Table II).

There is no clear evidence for a structural transformation down to $1.5 \mathrm{~K}$, although additional diffraction peaks appear below $T_{N}$ due to a magnetic transition, as discussed later. Figure 2(b) shows the refinement result for the diffraction pattern recorded at $1.5 \mathrm{~K}$. The reliability of fitting the data at $1.5 \mathrm{~K}$ is slightly worse than that at $200 \mathrm{~K}$, with a reduced $\chi_{\text {fit }}^{2}=5.34$ for $1.5 \mathrm{~K}$ and $\chi_{\text {fit }}^{2}=2.17$ for $200 \mathrm{~K}$. The quality of fit at $1.5 \mathrm{~K}$ is slightly improved by including a monoclinic distortion $\left[\beta=90.1(1)^{\circ}\right]$ (using the $\Gamma$ point subgroup $P 2_{1} / c$ or $P 2_{1} / m$ ). However, the obtained monoclinic distortion is too small to be firmly established with the resolution of the present NPD data. 


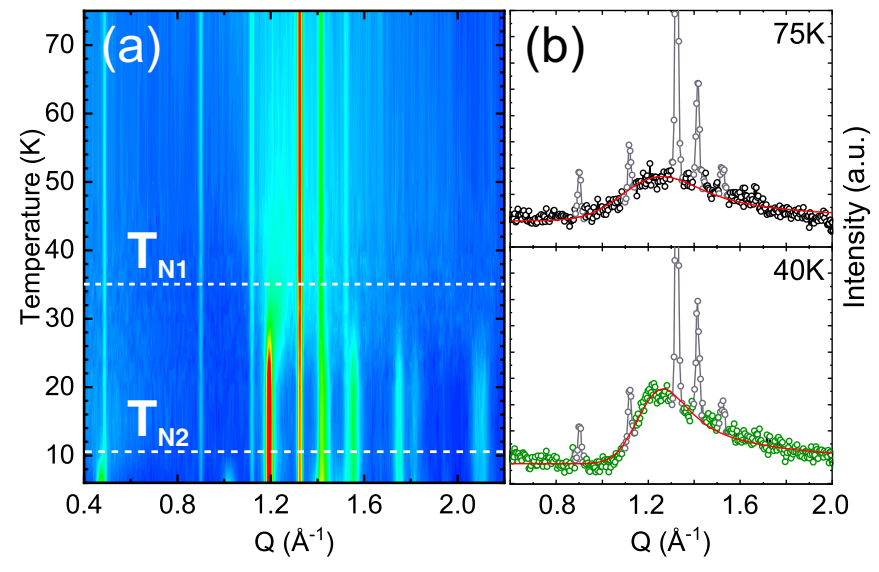

FIG. 4. (a) Temperature evolution of the powder neutron diffraction patterns of $\mathrm{NaMn}_{2} \mathrm{O}_{4}(\mathrm{DMC} \lambda=2.45 \AA$ ) between 75 and $6 \mathrm{~K}$ in the $Q=0.4-2.2 \AA^{-1}$ range. Here $T_{N 1} \approx 35 \mathrm{~K}$ and $T_{N 2} \approx 11 \mathrm{~K}$ are marked by the horizontal dashed white lines. (b) Neutron diffraction patterns at two temperatures above $T_{N 1} \approx 35 \mathrm{~K}$ in $\mathrm{NaMn}_{2} \mathrm{O}_{4}$. Circles show experimental data, Bragg peaks corresponding to the crystal structure are masked in gray, and the solid red line is a fit of the diffuse magnetic scattering to a Warren line shape given by [33].

Therefore, the low-temperature crystal structure is considered to be the same as the one at $200 \mathrm{~K}$ (Pnam). This is also supported by the results shown in Tables I and II, where the unit cell volume is found to contract only by $0.40(1) \%$, when temperature is decreased from $200 \mathrm{~K}$ to $1.5 \mathrm{~K}$. This means that both the charge ordering and orbital pattern remain unchanged at the onset of magnetic order. A possible structural transition induced by magnetic ordering will be discussed in the next section.

\section{B. Magnetic order of $\mathrm{NaMn}_{2} \mathrm{O}_{4}$ : Diffuse scattering}

In order to resolve and further understand the magnetic transition of $\mathrm{NaMn}_{2} \mathrm{O}_{4}$ [23], we have also performed NPD using the cold neutron powder diffractometer DMC (at PSI) as a function of temperature between 6 and $200 \mathrm{~K}$ [Fig. 4(a)]. The absence of three-dimensional long-range magnetic order is confirmed by the lack of magnetic diffraction peaks above $40 \mathrm{~K}$. However, a diffuse scattering is clearly observed, indicating the formation of short-range spin ordering. The shape of such magnetic diffuse scattering is asymmetric with a maximum at $Q \sim 1.2 \AA^{-1}$ and is well fitted with a Warren function [33] [see Fig. 4(b)]. This is characteristic of a 2D short-range order. In fact, the fit provides that, as temperature decreases from $75 \mathrm{~K}$ to $40 \mathrm{~K}$, the $2 \mathrm{D}$ correlation length increases from $\sim 18 \AA$ to $\sim 28 \AA$. Such a correlation length is comparable to those of closely related compounds with a similar diffraction profile, e.g., $\mathrm{NaMnO}_{2}$ [34] and $\mathrm{CuMn}_{2} \mathrm{O}_{4}$ [35] having a layered transition metal triangular lattice structure, as well as $\mathrm{Dy}_{2} \mathrm{TiO}_{5}$ [36] with a Dy triangular ladder configuration. Moreover, the previous $\mu^{+} \mathrm{SR}$ studies of $\mathrm{NaMn}_{2} \mathrm{O}_{4}$ [23] revealed the presence of a disordered state at temperatures between $T_{N}$ and $\sim 60 \mathrm{~K}$, which is fully consistent with the current NPD results. A more detailed interpretation of this diffuse scattering is given below in Sec. III C.

\section{Magnetic order of $\mathrm{NaMn}_{2} \mathrm{O}_{4}$ : Refinement of spin structure}

As temperature decreases from $40 \mathrm{~K}$, the magnetic diffuse scattering is gradually transformed into magnetic Bragg peaks [see Fig. 4(a)], which is consistent with the previous $\mu^{+} \mathrm{SR}$ result. Furthermore, there are clearly two magnetic transitions, $T_{N 1}=35 \mathrm{~K}$ and $T_{N 2}=11 \mathrm{~K}$. It is noteworthy that the $\chi(T)$ curve [Fig. 1(a)] shows the clear cusp of the AFM transition around $\sim 15 \mathrm{~K}$, which is much lower than the first $T_{N}$ determined by both $\mu^{+} \mathrm{SR}$ and NPD. Indeed, as seen in the differential susceptibility $(d \chi / d T)(T)$ curves [Fig. 1(b)], not only $T_{N 2}$ is well evidenced $(\sim 9 \mathrm{~K})$, but a small anomaly is also observed around $T_{N 1}(\sim 40 \mathrm{~K})$ in both zero-field-cooled and field-cooled modes (only the zero-field-cooled mode is shown). This is in perfect agreement with the observation of diffuse scattering in the NPD data. Such a vague anomaly observed around $T_{N 1}$ is rather abnormal, considering that clear magnetic Bragg peaks are observed by NPD. Such behavior might be related to the crystallinity (grain size) of the sample, as reported on Ref. [37].

Figure 5(a) compares the diffraction patterns recorded at $T=40,15$, and $6 \mathrm{~K}$ in order to clarify the change in Bragg peaks across $T_{N 1}$ and $T_{N 2}$. The difference between diffraction patterns at 40 and $15 \mathrm{~K}$ [Fig. 5(b)] clearly demonstrates the appearance of a set of magnetic Bragg peaks, which are all indexed by a commensurate (C) propagation vector $k_{\mathrm{C}}=$ $(0.5,0.5,0.5)$. On the other hand, the difference between the patterns at 15 and $6 \mathrm{~K}$ is instead indexed using an incommensurate (IC) propagation vector $k_{\mathrm{IC}}=(0,0,0.216(1))$. Surprisingly, despite the clear magnetic transition from the C-AFM phase to the IC-AFM phase at $T_{N 2}$, the C-AFM diffraction pattern does not change even below $T_{N 2}$ [see details in Fig. 5(b)]. This means that the C-AF structure is preserved even inside the IC-AF ordered state appeared below $T_{N 2}$. This also implies that these two magnetic sublattices are mutually independent. Based on this fact, each type of magnetic order is therefore predicted to be associated with only one crystallographic $\mathrm{Mn}$ site, either $\mathrm{Mn}^{3+}$ or $\mathrm{Mn}^{4+}$.

During the refinement process, many different magnetic models were attempted to fit the NPD data. To constrain the number of solutions for the magnetic models, symmetry analysis was performed using $k_{\mathrm{C}}$ and $k_{\mathrm{IC}}$ for each Wyckoff site $4 e$ of $\mathrm{Mn}$. The commensurate $k_{\mathrm{C}}$ ordering is described as C-AFM chains running along the $c$ axis $\left(J_{2}\right)$. Next-neighbor $\mathrm{Mn}$ sites arrange antiferromagnetically along [01 - 1] and ferromagnetically along [011] $\left(J_{1}\right)$. In other words, the Mn spin chains make a -[AFM-FM-AFM-FM]- zigzag pattern along the $c$ axis (see Fig. 7). The proposed model is expressed by the $C_{a} c$ Shubnikov group (9.41 in Belov-Neronova-Smirnova notation), which has four independent magnetic $\mathrm{Mn}$ sites derived from each Mn site (a split of the $4 c$ sites into two orbits) within this magnetic symmetry. Since both sites are crystallographically identical, we can simplify the refinement process by assuming that they also acquire the same ordered magnetic moment. Using this spin arrangement, the diffraction pattern recorded at $15 \mathrm{~K}$ is well fitted with only one $\mathrm{Mn}$ site [Fig. 5(c)], leading to the best $R_{\mathrm{Bragg}}=1.9 \%$ and $R_{\mathrm{mag}}=$ $8.4 \%$. Such a high $R_{\text {mag }}$ is mainly related to the remnant of diffuse scattering on the shoulder of several magnetic Bragg peaks, especially for the one at $Q \sim 1.2 \AA^{-1}$. 


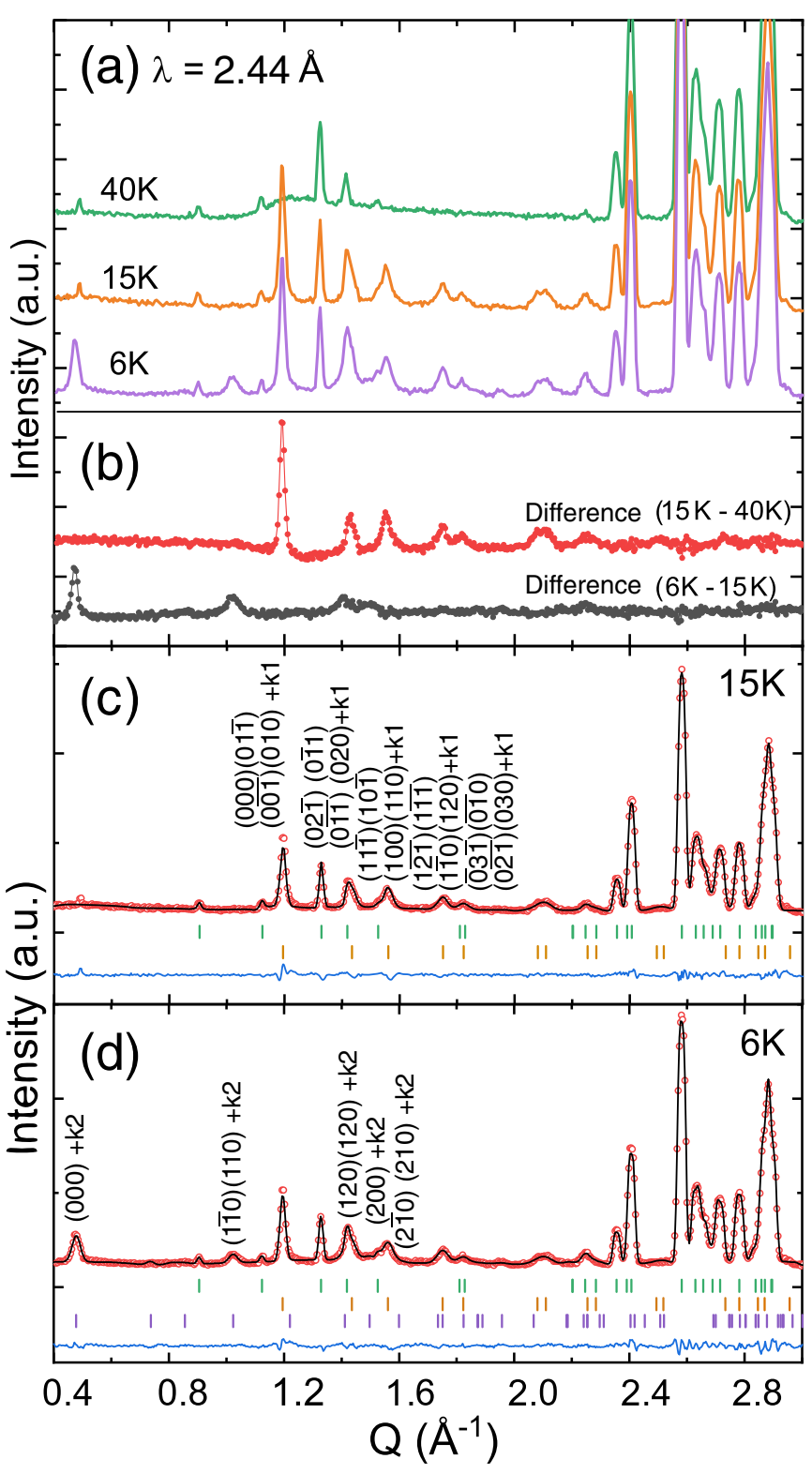

FIG. 5. (a) Neutron powder diffraction patterns recorded at $T=$ 6,15 , and $40 \mathrm{~K}$ and (b) their respective differences (acquired using a DMC instrument using $\lambda=2.45 \AA$ ). Also shown is the Rietveld refinement of the neutron diffraction data of $\mathrm{NaMn}_{2} \mathrm{O}_{4}$ at (c) $15 \mathrm{~K}$ and (d) $6 \mathrm{~K}$. Green, orange, and purple ticks indicate Bragg peak positions of crystal, commensurate magnetic, and incommensurate magnetic structures, respectively.

To conduct refinement using both $\mathrm{Mn}^{3+}$ and $\mathrm{Mn}^{4+}$ can also fit the NPD data sufficiently. However, one Mn site shows a negligible size of the magnetic moment with a large error. Thus the final refinement was performed using only one Mn site. This result clearly also supports our hypothesis with two independent magnetic sublattices, each consisting of only either $\mathrm{Mn}^{3+}$ or $\mathrm{Mn}^{4+}$. Using only one $\mathrm{Mn}$ site, the Rietveld refinement [Fig. 5(c)] yields $m_{y}=2.6(1)$ and $m_{z}=0.4(1)$, which gives a total ordered magnetic moment $\mu_{\text {ord }}^{\mathrm{C}}=2.63(2) \mu_{\mathrm{B}}$ at $T=15 \mathrm{~K}$.

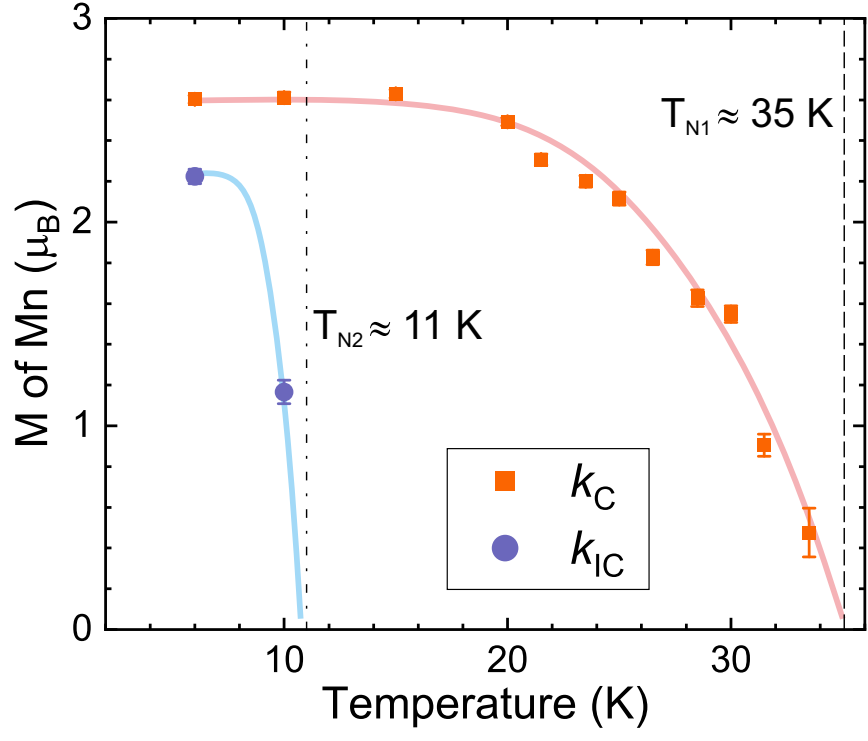

FIG. 6. Temperature evolution of the $\mathrm{Mn}^{3+}$ and $\mathrm{Mn}^{4+}$ ordered magnetic moments (from Rietveld refinement results). Solid lines are guides to the eye, while the dashed line and dash-dotted line are at $T_{N 1}=35 \mathrm{~K}$ and $T_{N 2}=11 \mathrm{~K}$, respectively.

It should be noted that $m_{x}$ is set to zero even though it is not restricted by symmetry. This is because we found that refining this component did not improve the fitting of our NPD data. On the other hand, the small component $m_{z}$ is necessary to obtain a better fit for the magnetic Bragg peaks. The size of $\mu_{\mathrm{ord}}^{\mathrm{C}}$ remains unchanged down to $6 \mathrm{~K}$ (Fig. 6). The main component of $\mu_{\text {ord }}^{\mathrm{C}}$, i.e., $m_{y}$, in the two adjacent chains follows approximately the orbital pattern of $\mathrm{Mn}^{3+}$ as described earlier. This spin direction is understandable by considering the single-ion anisotropy of $\mathrm{Mn}^{3+}$ in the elongated octahedra along the local $z$ axes due to the Jahn-Teller distortions. However, the spin alignment slightly deviates $\left(\sim 14^{\circ}\right)$ from the local $z$ orbital. Such deviation possibly results from the competition between magnetic anisotropy, frustration and $90^{\circ}$ superexchange interaction.

For the incommensurate $k_{\mathrm{IC}}$ order, there are four onedimensional irreducible representations and three basis functions for each irreducible representation. Only the symmetry modes spanned by the irreducible representations $\Gamma_{4}$ provide good agreement with the experimental data. Table III lists the basis vectors of irreducible representations $\Gamma_{4}$, obtained by the projection operator method. The low-temperature IC-AFM order was first fitted using only one Mn site, following the C-AFM phase results. Figure 7 shows the spin structure that gives the factor $R_{\mathrm{mag}}=13.6 \%$ in the best agreement with the experimental data (see Fig. 5). This model corresponds to a spin-density wave (SDW), obtained by mixing the basis function $\psi_{1}$ and $\psi_{3}$ of real components only. At $6 \mathrm{~K}, m_{x}=1.4(1)$ and $m_{z}=1.5(1)$ are obtained by a refinement of the SDW magnetic structure [Fig. 5(d)]. For this model, the maximum amplitude of the SDW is about 2.0(1) $\mu_{\mathrm{B}}\left(=\mu_{\text {ord }}^{\mathrm{IC}}\right)$. Here $\mu_{\text {ord }}^{\mathrm{IC}}$ lies in the $a c$ plane with the spin modulation in the [101] and $[10-1]$ directions. The intraleg interaction is AFM with the domain boundary shown as the dotted line in Fig. 7(c). 
TABLE III. Basis functions for axial vectors associated with the irreducible representation $\Gamma_{4}$ for Wyckoff site $4 c$ and $k_{\mathrm{IC}}=(0,0,0.216)$. Here $a=e^{+i \pi q}$

\begin{tabular}{lcccc}
\hline \hline & Mn2-1 & Mn2-2 & Mn2-3 & Mn2-4 \\
$\Gamma_{4}$ & $(x y z)$ & $\left(-x+1,-y+1, z+\frac{1}{2}\right)$ & $\left(-x+\frac{1}{2}, y-\frac{1}{2}, z+\frac{1}{2}\right)$ & $\left(x+\frac{1}{2},-y+\frac{3}{2}, z\right)$ \\
\hline$\psi_{1}$ & $(100)$ & $\left(a^{*} 00\right)$ & $\left(a^{*} 00\right)$ & $(100)$ \\
$\psi_{2}$ & $(010)$ & $\left(0 a^{*} 0\right)$ & $\left(0-a^{*} 0\right)$ & $(0-10)$ \\
$\psi_{3}$ & $(001)$ & $\left(00-a^{*}\right)$ & $\left(00-a^{*}\right)$ & $(001)$ \\
\hline \hline
\end{tabular}

Indeed, the IC-AFM Bragg peaks can also be fitted using a cycloidal magnetic modulation (instead of the SDW described above). Such a model is obtained by mixing the basis functions $\psi_{1}$ and $\psi_{3}$ using real and imaginary components. The magnetic moments in this model also lie in the $a c$ plane. At $6 \mathrm{~K}$, the components of the moment are $m_{x}=1.5(1)$ and $m_{z}=0.3(2)$ and the ordered component maximum is 1.5 $\left(=m_{x}\right)$. The agreement factor using the cycloidal model is

(a)

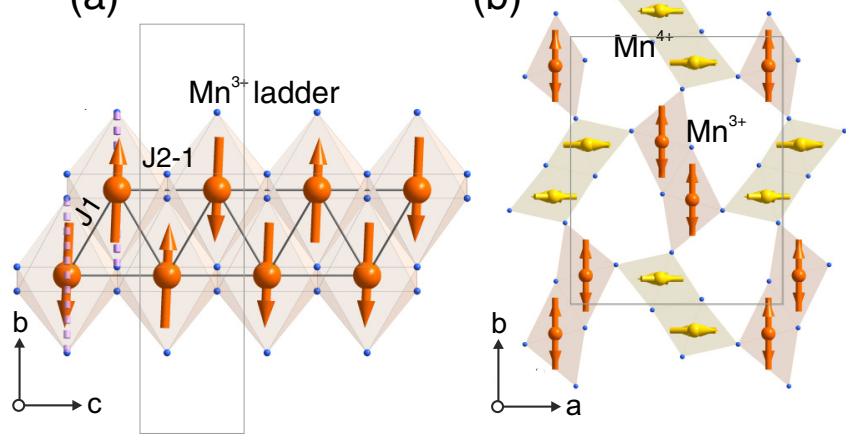

(c)

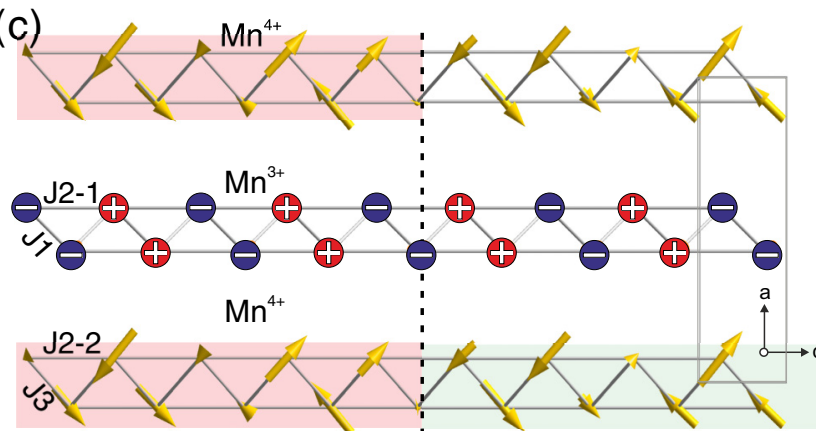

(d)

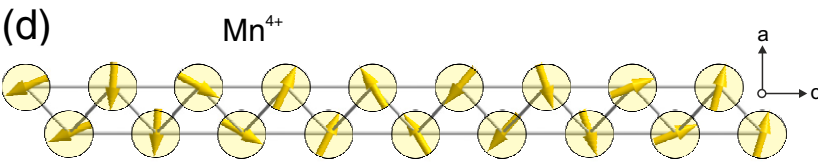

FIG. 7. Magnetic structure of $\mathrm{NaMn}_{2} \mathrm{O}_{4}$ at $6 \mathrm{~K}$. (a) The $k_{\mathrm{C}}$ commensurate spin configuration along the $\mathrm{Mn}^{3+}$ ladder. The purple dashed line is a guide to indicate the direction of the $z$ orbital. (b) Overall spin structure of $\mathrm{NaMn}_{2} \mathrm{O}_{4}$ along the $c$ axis in the honeycomb mesh. (c) Two Mn ladders along the $c$ axis. The dashed line highlights the spin inversion plane of the $k_{\mathrm{IC}}$ incommensurate spin arrangement. The magnetic moments of commensurate spins along in the $b$ direction are symbolized by + or - signs depending on their spin direction. (d) Single Mn ladder showing the alternative cycloidal incommensurate spin arrangement (projected as elliptical in the $a c$ plane).
$R_{\text {mag }}=18.6 \%$, which is slightly higher than one obtained for the SDW model. For both models, the periodicity of the IC modulation is $\sim 4.63 c$ and the incommensurate spin structures are also described by $\psi_{2}$ (corresponding to the $m_{y}$ component) based on the symmetry analysis. An unconstrained refinement using DMC data $(\lambda=2.45 \AA)$, using only the symmetry restrictions of $\Gamma_{4}$, show that the $m_{y}$ component of $\mu_{\text {ord }}^{\mathrm{IC}}$ is nearly zero. As a result, in the final refinement stage, the $m_{y}$ component is therefore set to zero.

Because $\mu_{\text {ord }}^{\mathrm{C}}$ is greater than $\mu_{\text {ord }}^{\mathrm{IC}}$, the $k_{\mathrm{C}}$ magnetic structure is assumed to be of the $\mathrm{Mn}^{3+}(\mathrm{Mn} 1)$ site while the $k_{\mathrm{IC}}$ phase is for $\mathrm{Mn}^{4+}(\mathrm{Mn} 2)$. Both magnetic moments are smaller than the expected values for the fully ordered state (for $\mathrm{Mn}^{3+}$, $S=2$ and $4.9 \mu_{\mathrm{B}}$, and for $\mathrm{Mn}^{4+}, S=3 / 2$ and $3.8 \mu_{\mathrm{B}}$ ), which is coherent with the remnant diffuse scattering. Such an effect is also reported for, e.g., $\mathrm{NaMnO}_{2}\left(2.92 \mu_{\mathrm{B}}\right.$ for $\left.\mathrm{Mn}^{3+}\right)$ [34]. It is also worthwhile to mention the possibility of a slight intermixing between $\mathrm{Mn}^{3+}$ and $\mathrm{Mn}^{4+}$ (both $\mathrm{Mn}$ sites can form $\mathrm{C}$-AFM and IC-AFM configuration). This is a well-known issue in charge ordered manganites and such mixing often leads to smaller $\mu_{\text {ord }}$ than the expected value of, e.g., $\mathrm{NaMn}_{7} \mathrm{O}_{12}$ $\left(2.85 \mu_{\mathrm{B}}\right.$ for $\mathrm{Mn}^{3+}$ and $2.40 \mu_{\mathrm{B}}$ for $\mathrm{Mn}^{4+}$ at $\left.10 \mathrm{~K}\right)$ [38].

For the asymmetric diffuse scattering around the commensurate magnetic peaks at temperature above $T_{N 1}$, the strongest intensity was observed at $Q \sim 1.2 \AA^{-1}[(000)(01-1)(00-$ $\left.\left.1)(010)+k_{\mathrm{C}}\right)\right]$ (see Sec. III B). This position suggests that the short-range correlations are initiated along the chain axis (bc plane), which is compatible with the expected magnetic interaction along this direction (i.e., $J_{2-1}$, corresponding to the commensurate ordering).

The details of the $k_{\mathrm{C}}$ magnetic structure are described in Fig. 7, with the antiferromagnetic chains parallel to the $c$ axis. All the intraleg Mn-O-Mn supercharge interactions are rather weak, because the angle of the $\mathrm{Mn}-\mathrm{O}-\mathrm{Mn}$ bonds is nearly $90^{\circ}$. However, for the case of the nearest-neighbor interaction $\left(J_{2}\right)$, both $90^{\circ} \mathrm{Mn} 1-\mathrm{O} 1-\mathrm{Mn} 1$ and Mn1-O4-Mn1 bonds are formed by a direct overlap of the $d_{x y}$ (or $d_{y z}$ or $d_{z x}$ ) orbitals. Consequently, strong AFM direct-exchange interactions are possible [29], which causes a geometrically frustrated configuration. Due to such frustration on the triangular lattice in the two-leg ladder, a magnetoelastic coupling is expected to appear below $T_{N 1}$. The $C_{a} c$ Shubnikov group has a monoclinic supercell with $a_{\mathrm{m}} \sim 5.68 \AA, b_{\mathrm{m}} \sim 17.7 \AA, c_{\mathrm{m}} \sim 11.5 \AA$, and $\beta_{\mathrm{m}} \sim 104^{\circ}$. In this supercell, there is a $\Gamma^{3+}$ displacement mode [with $k$ vector $(0,0,0)$ and direction acorresponding to the isotropy subgroup $P 2_{1} / c$ (No. 14)]. This allows a shift of $\mathrm{Mn}$ atoms in the $c$ direction, but two Mn rows (within the ladders) then shift in the opposite $z$ directions. This distortion component could deform the triangular lattices in order to stabilize the $J_{1}$ magnetic interaction. However, as mentioned 
in Sec. III A, the monoclinic distortion in the crystal structure is not evident in the present NPD data. Further studies at low temperatures using high-resolution single-crystal synchrotron $\mathrm{x}$-ray- and electron-diffraction techniques will be valuable for investigating the details of a magnetoelastic coupling in the $\mathrm{NaMn}_{2} \mathrm{O}_{4}$ compound.

For the $k_{\mathrm{IC}}$ (IC-AFM) magnetic structure, we have here proposed both a SDW and an alternative cycloidal model. The coupling within the $\mathrm{Mn}^{4+}$ ladder is rather complicated to interpret and also it is impossible to distinguish between these models using only the powder diffraction method. The cycloidal model is more reasonable in a uniform valence state system. A uniform valence state $\mathrm{CFO}-\mathrm{CaCr}_{2} \mathrm{O}_{4}$ has been reported to show the cycloidal magnetic order with the IC propagation vector $k=(0,0,0.477)$ and the magnetic moments lie in the $a c$ plane [5]. On the other hand, one could compare with mixed valence $\mathrm{CFO}-\mathrm{NaV}_{2} \mathrm{O}_{4}$, which poses a SDW magnetic structure similar to the IC propagation vector $k=(0,0.19,0)[4]$. The direction of the ordered spins was found to be perpendicular to the ladder direction with the SDW modulation along the ladder. This configuration and modulation are similar to the current $\mathrm{Mn}^{4+}$ ladder case, except that $\mathrm{NaMn}_{2} \mathrm{O}_{4}$ has magnetic components (roughly $\sim 30^{\circ}$ ) along the ladder direction. This might be due to the additional superexchange interaction (intermediate angle $\sim 130^{\circ}$ ) between $\mathrm{Mn}^{3+}$ and $\mathrm{Mn}^{4+}$ ladders. In $\mathrm{NaMn}_{2} \mathrm{O}_{4}$, we assume that the $\mathrm{Mn}^{3+}$ and $\mathrm{Mn}^{4+}$ are perfectly ordered and the IC-AFM phase only involves $\mathrm{Mn}^{4+}$ spin ordering. Thus, the IC-AFM phase can be considered a uniform valence state system of $\mathrm{Mn}^{4+}$, which would support the cycloidal model. However, the SDW model cannot be completely ruled out since there is a possibility of slight disordering between $\mathrm{Mn}^{3+}$ and $\mathrm{Mn}^{4+}$. In order to understand the detail of such spin coupling, further magnetic characterization will be required, such as singlecrystal studies and inelastic neutron scattering, along with theoretical calculations and computer simulations. Finally, it could also be very interesting to perform additional investigations under high hydrostatic pressure to know how subtle, externally induced structural distortions affect the magnetic correlations [39] in this complex material.

\section{Neutron diffraction study of $\mathrm{Li}_{0.92} \mathrm{Mn}_{2} \mathrm{O}_{4}$}

We have also performed a NPD study on the closely related CFO-type compound $\mathrm{Li}_{0.92} \mathrm{Mn}_{2} \mathrm{O}_{4}$ in order to compare the magnetic nature within this family. The crystal structure of $\mathrm{Li}_{0.92} \mathrm{Mn}_{2} \mathrm{O}_{4}$ was confirmed as a reported one [22]. Unlike $\mathrm{NaMn}_{2} \mathrm{O}_{4}, \mathrm{Li}_{0.92} \mathrm{Mn}_{2} \mathrm{O}_{4}$ lacks a clear charge and/or orbital order; thus both $\mathrm{Mn}^{3+}$ and $\mathrm{Mn}^{4+}$ are equally and randomly distributed on the two Mn sites. The resolution and $Q$ range of the present neutron diffraction study recorded only using the cold neutron DMC diffractometer $(\lambda=2.45 \AA)$ are not enough to perform a proper structural analysis. Therefore, the Rietveld refinement was performed using reference structural parameters [15], yielding a reasonable fit result. The temperature-dependent data revealed an absence of any changes in the crystal structure for temperatures between 1.5 and $120 \mathrm{~K}$. Moreover, the NPD result revealed the presence of the $\mathrm{Li}_{2} \mathrm{MnO}_{3}$ impurity phase with a volume fraction of $16.1 \%$. This is in good agreement with the previously

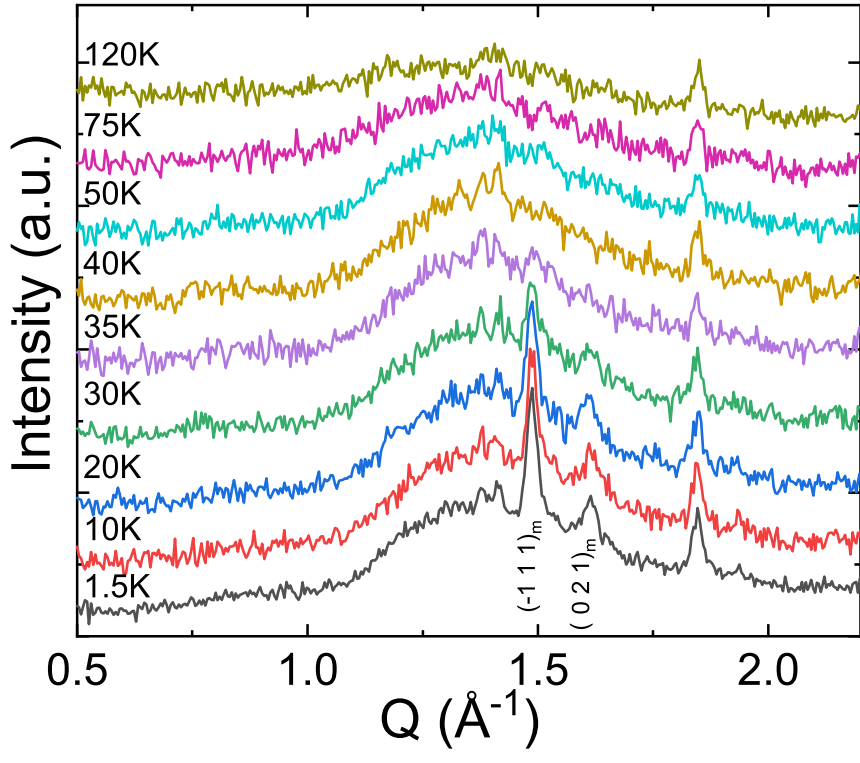

FIG. 8. Temperature evolution of the powder neutron diffraction patterns of $\mathrm{Li}_{0.92} \mathrm{Mn}_{2} \mathrm{O}_{4}$ (DMC $\lambda=2.45 \AA$ ) between 1.5 and $120 \mathrm{~K}$ in the $Q=0.5-2.2 \AA^{-1}$ range. Two magnetic Bragg peaks of the $\mathrm{Li}_{2} \mathrm{MnO}_{3}$ impurity phase are indexed.

reported XRD and $\mu^{+} \mathrm{SR}$ results [15,23]. From the previous $\mu^{+} \mathrm{SR}$ measurement of $\mathrm{Li}_{0.92} \mathrm{Mn}_{2} \mathrm{O}_{4}$, the observed magnetic signal was in fact found to come from the $\mathrm{Li}_{2} \mathrm{MnO}_{3}$ phase [23]. The present NPD results are fully consistent with the reported $\mu^{+} \mathrm{SR}$ data; in other words, only magnetic Bragg peaks corresponding to the $\mathrm{Li}_{2} \mathrm{MnO}_{3}$ phase were observed below $T_{N}=35 \mathrm{~K}$ [40]. Two main magnetic Bragg peaks of $\mathrm{Li}_{2} \mathrm{MnO}_{3}$ are indexed and shown in Fig. 8. There is also clear diffuse scattering (maximum around $Q \sim 1.3 \AA^{-1}$ ) correlated with a background reduction, which indicates the appearance of short-range magnetic order. Below $50 \mathrm{~K}$, such diffuse scattering remains unchanged down to $1.5 \mathrm{~K}$, i.e., even below the magnetic transition temperature of $\mathrm{Li}_{2} \mathrm{MnO}_{3}$. This suggests that the diffuse scattering is not originating from $\mathrm{Li}_{2} \mathrm{MnO}_{3}$ but instead is an intrinsic feature of the $\mathrm{Li}_{0.92} \mathrm{Mn}_{2} \mathrm{O}_{4}$ compound, which has a magnetic transition at $T_{N}=44 \mathrm{~K}$ according to the $\mu^{+}$SR results [23]. Further studies, probably at even lower temperatures, will be required to obtain further information on the detailed magnetic nature of $\mathrm{Li}_{0.92} \mathrm{Mn}_{2} \mathrm{O}_{4}$.

\section{DISCUSSION}

$\mathrm{NaMn}_{2} \mathrm{O}_{4}$ is found to exhibit two successive antiferromagnetic transitions at $T_{N 1}=35 \mathrm{~K}$ and $T_{N 2}=11 \mathrm{~K}$. The former is a transition to a $\mathrm{C}$-AFM long-range $\mathrm{Mn}^{3+}$ spin ordered state and the latter is a transition to an IC-AFM $\mathrm{Mn}^{4+}$ spin ordered state (SDW or cycloidal). Our study indicates that these two $\mathrm{Mn}^{3+} / \mathrm{Mn}^{4+}$ magnetic lattices are independent of each other. The inverse susceptibility $1 / \chi$, shown in Fig. 1(c), is linear with temperature above $200 \mathrm{~K}$; however, it deviates strongly from such a linear relationship below around $150 \mathrm{~K}$. This is much higher than the clear AFM cusp found at around $15 \mathrm{~K}$. If the temperature evolution of the Mn moment in the $\mathrm{Mn}^{3+}$ sublattice is independent of that in the $\mathrm{Mn}^{4+}$ sublattice, each magnetization follows a Curie-Weiss behavior with its 
own Curie constant $C$ and Weiss temperature $\Theta$. Since $\Theta$ of the $\mathrm{Mn}^{3+}$ sublattice is naturally different from that of the $\mathrm{Mn}^{4+}$ sublattice, such a difference causes nonlinearity of the $1 / \chi(T)$ curve. This could be a reason why the deviation appears already at temperatures as high as $150 \mathrm{~K}$. Above $200 \mathrm{~K}$, the difference of $C$ and $\Theta$ between two sublattices becomes relatively small compared to the temperature, resulting in the linear relationship between $1 / \chi$ and temperature.

In order to understand the complex and unique magnetic ground state of $\mathrm{NaMn}_{2} \mathrm{O}_{4}$, we further compare the series of $A \mathrm{Mn}_{2} \mathrm{O}_{4}(A=\mathrm{Li}, \mathrm{Na}$, and $\mathrm{Ca})$. Note that the crystal structure of JT-ordered $\mathrm{CaMn}_{2} \mathrm{O}_{4}$ consists of double-rutile chains, which are connected through edge-sharing oxygen atoms. This structure is slightly different from the CFO-type structure, in which the chains are interconnected through corner-sharing oxygen atoms instead [3]. Furthermore, the $\mathrm{CaMn}_{2} \mathrm{O}_{4}$ structure has a $180^{\circ}$ superexchange path so that the Heisenberg exchange interactions have priority over the easy axis anisotropy. As a result, the spin direction is independent of the orbital ordering pattern (the spins are parallel to the ladders). The magnetic ground state of $\mathrm{NaMn}_{2} \mathrm{O}_{4}$ is rather unique, because each of the two different Mn sites has its own ordering temperature and modulation. Although no detailed calculation has been reported so far, the interaction between $\mathrm{Mn}^{3+}$ and $\mathrm{Mn}^{4+}$ ladders (in the $a b$ plane, $J_{4}$ and $J_{5}$ ) are most likely much weaker than the interaction within each individual ladder $\left(J_{1}, J_{2}\right.$, and $\left.J_{3}\right)$. This is evidenced by the spin configuration of $\mathrm{Mn}^{3+}$ not being affected by the onset of $\mathrm{Mn}^{4+}$ spin order. Within the $\mathrm{Mn}^{3+}$ ladder, the single-ion anisotropy dominates owing to superimposition of the $3 d$ electrons in the $d_{z^{2}}$ orbitals of $\mathrm{Mn}^{3+}$. On the other hand, in the $\mathrm{Mn}^{4+}$ ladders, there is no contribution from anisotropy but the competing exchange interaction is favored instead (because of the absence of the $d_{z^{2}}$ orbital order). The combination of the complex exchange pathways, i.e., edge and corner-sharing octahedra through the oxygen, and geometrical frustration on the triangular lattice then often result in a complex incommensurate spin structure, such as $\mathrm{CFO}-\mathrm{NaV}_{2} \mathrm{O}_{4}$ and $\mathrm{CaCr}_{2} \mathrm{O}_{4}$ $[4,5]$.

As for $\mathrm{Li}_{0.92} \mathrm{Mn}_{2} \mathrm{O}_{4}$, no long-range magnetic order was observed down to $1.5 \mathrm{~K}$ by the present neutron powder diffraction experiment. This is most likely due to the nonstoichiometry of $\mathrm{Li}$, which naturally increases the amount of $\mathrm{Mn}^{4+}$ by about $8 \%$ from that $(50 \%)$ of $\mathrm{NaMn}_{2} \mathrm{O}_{4}$. As a result, it is impossible to form the ladder occupied only by $\mathrm{Mn}^{3+}$ but likely to form the two ladders with random distributions of $\mathrm{Mn}^{3+}$ and $\mathrm{Mn}^{4+}$. This effectively hinders the formation of long-range magnetic order down to at least $1.5 \mathrm{~K}$.

It is therefore concluded that charge and orbital order play a significant role for the alignment of the $\mathrm{Mn}$ moments in the triangular spin ladders. This also suggests the possibility that the magnetic ground state of $\mathrm{NaMn}_{2} \mathrm{O}_{4}$ is sensitive to the $\mathrm{Na}$ content. Therefore, it would be very interesting to study $\mathrm{Na}_{1-\delta} \mathrm{Mn}_{2} \mathrm{O}_{4}$ prepared by an electrochemical reaction with neutron scattering and $\mu^{+} \mathrm{SR}$.

The spin-orbital coupling in JT active manganites has been reported for several compounds. Typical examples are the manganese perovskites $L n \mathrm{MnO}_{3}$, where $\mathrm{Ln}=\mathrm{La}$ to $\mathrm{Gd}$ and Tl [41,42]. Such compounds show noncollinear orbital order with collinear spin order, owning to the competition between single anisotropy and exchange interactions. The compound series of $A_{1-x} A_{x}^{\prime} \mathrm{MnO}_{3}\left(A=\mathrm{La}\right.$ and $A^{\prime}=\mathrm{Ca}, \mathrm{Sr}$, and $\left.\mathrm{Ba}\right)$ is known to have charge, orbital, and magnetic order. Similar to $\mathrm{NaMn}_{2} \mathrm{O}_{4}$, the spin structure of $\mathrm{La}_{0.5} \mathrm{Ca}_{0.5} \mathrm{MnO}_{3}$ consists of two different magnetic sublattices, which are also based on $\mathrm{Mn}^{3+}$ and $\mathrm{Mn}^{4+}$ sites [43]. Besides the perovskites, very recently, the layered honeycomb $\mathrm{BiMnTeO}_{6}$ was reported to show the noncollinear spin-orbital coupling [44].

In comparison, layered triangular lattice compounds such as $\mathrm{CuMnO}_{2}$ and $\mathrm{NaMnO}_{2}$ pose a frustrated triangular spin lattice with strong anisotropic interaction due to the ferro-orbital ordering. Such magnetic frustration leads to a magnetoelastic coupling, evidenced by the structural transition [34,45]. Moreover, several studies concerning the correlation between spin and orbital order were reported for $\mathrm{CaMn}_{7} \mathrm{O}_{12}$, which reveal that both crystal (orbital) and magnetic (spin) structures are incommensurate. Such unique modulation plays a role in breaking the inversion symmetry, resulting in one of the best type-II multiferroic materials [46,47]. A better and much more detailed understanding of spin and orbital order in these compounds would also be very useful for various technical applications [48]. $\mathrm{NaMn}_{2} \mathrm{O}_{4}$ is therefore an intriguing example of a charge-orbital-spin correlated manganites. In particular, inelastic neutron scattering measurements using a single-crystal sample together with theoretical work will be extremely valuable to further understand the detailed relationship between orbital physics and magnetism not only in manganites but also for the general group of transition metal oxides.

\section{CONCLUSION}

This study of CFO-NaMn${ }_{2} \mathrm{O}_{4}$ has revealed a complex magnetic ground state, based on successive charge, orbital, and spin order. Based on high-resolution neutron powder diffraction, the $\mathrm{Mn}^{3+}$ and $\mathrm{Mn}^{4+}$ were found to be fully ordered at the lowest temperature $(T=1.5 \mathrm{~K})$. Although $2 \mathrm{D}$ shortrange spin order presents already at $75 \mathrm{~K}$, a commensurate antiferromagnetic ordered phase appears below $T_{N 1}=35 \mathrm{~K}$ and then an incommensurate antiferromagnetic ordered phase follows below $T_{N 2}=11 \mathrm{~K}$. Surprisingly, the two AFM orders are found to be independent of each other. The C-AFM ordering is based on $\mathrm{Mn}^{3+} \mathrm{AFM}$ coupling along the chain axis, while the IC-AFM ordering is a SDW or cycloidal order residing on the $\mathrm{Mn}^{4+}$. Finally, neutron diffraction studies of the closely related $\mathrm{Li}_{0.92} \mathrm{Mn}_{2} \mathrm{O}_{4}$ compound clarify the absence of long-range order down to $1.5 \mathrm{~K}$. The existence of a diffuse scattering below $50 \mathrm{~K}$, however, clearly indicates the presence of short-range magnetic correlations in $\mathrm{Li}_{0.92} \mathrm{Mn}_{2} \mathrm{O}_{4}$.

\section{ACKNOWLEDGMENTS}

The authors thank H. Nozaki (Toyota Central Research and Development Labs., Inc.) for support during the neutron diffraction experiment. The neutron scattering measurements were performed using the DMC and HRPT instruments at the Swiss Spallation Neutron Source of the Paul Scherrer Institute in Villigen, Switzerland. The authors wish to thank the staff of PSI for the very valuable help with these experiments. This research was funded by the (Swedish Foundation 
for Strategic Research within the Swedish National Graduate School in Neutron Scattering as well as the Swedish Research Council (VR) through a neutron project grant (BIFROST, Grant No. 2016-06955). Further, Y.S. acknowledge funding from the (Swedish Research Council (VR) through a Starting Grant (No. 201705078) as well as Chalmers Area of
Advance-Materials Science; J.S. was supported by the Ministry of Education, Culture, Sports, Science and Technology of Japan, KAKENHI Grant No. 23108003, and Japan Society for the Promotion Science (JSPS) KAKENHI Grant No. JP18H01863; and H.S. acknowledges funding from JSPS KAKENHI Grant No. JP17K05521.
[1] T. Masuda, A. Zheludev, H. Manaka, L. P. Regnault, J. H. Chung, and Y. Qiu, Phys. Rev. Lett. 96, 047210 (2006).

[2] L. Mihály, B. Dóra, A. Ványolos, H. Berger, and L. Forró, Phys. Rev. Lett. 97, 067206 (2006).

[3] C. D. Ling, J. J. Neumeier, and D. N. Argyriou, J. Solid State Chem. 160, 167 (2001).

[4] H. Nozaki, J. Sugiyama, M. Månsson, M. Harada, V. Pomjakushin, V. Sikolenko, A. Cervellino, B. Roessli, and H. Sakurai, Phys. Rev. B 81, 100410(R) (2010).

[5] F. Damay, C. Martin, V. Hardy, A. Maignan, G. André, K. Knight, S. R. Giblin, and L. C. Chapon, Phys. Rev. B 81, 214405 (2010).

[6] C. Ling and F. Mizuno, Chem. Mater. 25, 3062 (2013).

[7] X. Liu, X. Wang, A. Iyo, H. Yu, D. Li, and H. Zhou, J. Mater. Chem. A 2, 14822 (2014).

[8] J. Akimoto, J. Awaka, N. Kijima, Y. Takahashi, Y. Maruta, K. Tokiwa, and T. Watanabe, J. Solid State Chem. 179, 169 (2006).

[9] D. Khomskii, Physics 2, 20 (2009).

[10] H. Kawamura, J. Phys.: Condens. Matter 10, 4707 (1998).

[11] H. Sakurai, T. Kolodiazhnyi, Y. Michiue, E. TakayamaMuromachi, Y. Tanabe, and H. Kikuchi, Angew. Chem. Int. Ed. 51, 6653 (2012).

[12] M. Taguchi, H. Yamaoka, Y. Yamamoto, H. Sakurai, N. Tsujii, M. Sawada, H. Daimon, K. Shimada, and J. Mizuki, Phys. Rev. B 96, 245113 (2017).

[13] H. Nozaki, H. Sakurai, O. Ofer, E. J. Ansaldo, J. H. Brewer, K. H. Chow, V. Pomjakushin, L. Keller, K. Prša, K. Miwa, M. Månsson, and J. Sugiyama, Physica B 551, 137 (2017).

[14] J. Sugiyama, H. Nozaki, M. Harada, Y. Higuchi, H. Sakurai, E. J. Ansaldo, J. H. Brewer, L. Keller, V. Pomjakushin, and M. Månsson, Phys. Proc. 75, 868 (2015).

[15] K. Mukai, T. Uyama, and I. Yamada, ACS Omega 4, 6459 (2019).

[16] M. Mamiya, K. Kataoka, J. Akimoto, S. Kikuchi, Y. Terajima, and K. Tokiwa, J. Power Sources 244, 561 (2013).

[17] M. Mamiya, K. Tokiwa, and J. Akimoto, J. Power Sources 310, 12 (2016).

[18] M. Månsson, I. Umegaki, H. Nozaki, Y. Higuchi, I. Kawasaki, I. Watanabe, H. Sakurai, and J. Sugiyama, J. Phys.: Conf. Ser. 551, 012035 (2014).

[19] I. Umegaki, H. Nozaki, M. Harada, M. Månsson, H. Sakurai, I. Kawasaki, I. Watanabe, and J. Sugiyama, JPS Conf. Proc. 21, 011018 (2018).

[20] K. Yamaura, M. Arai, A. Sato, A. B. Karki, D. P. Young, R. Movshovich, S. Okamoto, D. Mandrus, and E. TakayamaMuromachi, Phys. Rev. Lett. 99, 196601 (2007).

[21] K. Yamaura, Q. Huang, L. Zhang, K. Takada, Y. Baba, T. Nagai, Y. Matsui, K. Kosuda, and E. Takayama-Muromachi, J. Am. Chem. Soc. 128, 9448 (2006).
[22] K. Tokiwa, K. Matsukura, S. Kasahara, S. Tsuda, S. Mikusu, K. Takeuchi, A. Iyo, Y. Tanaka, J. Akimoto, J. Awaka, N. Kijima, Y. Takahashi, and T. Watanabe, J. Phys.: Conf. Ser. 150, 042210 (2009).

[23] J. Sugiyama, Y. Ikedo, O. Ofer, M. Månsson, E. J. Ansaldo, J. H. Brewer, K. H. Chow, H. Sakurai, and E. Takayama-Muromachi, J. Phys. Soc. Jpn. 78, 1 (2009).

[24] P. Fischer, G. Frey, M. Koch, M. Könnecke, V. Pomjakushin, J. Schefer, R. Thut, N. Schlumpf, R. Bürge, U. Greuter, S. Bondt, and E. Berruyer, Physica B 276-278, 146 (2000).

[25] J. Rodríguez-Carvajal, Physica B 192, 55 (1993).

[26] M. I. Aroyo, A. Kirov, C. Capillas, J. M. Perez-Mato, and H. Wondratschek, Acta Crystallogr. A 62, 115 (2006).

[27] E. Kroumova, M. I. Aroyo, J. M. Perez-Mato, A. Kirov, C. Capillas, S. Ivantchev, and H. Wondratschek, Phase Transit. 76, 155 (2003).

[28] B. J. Campbell, H. T. Stokes, D. E. Tanner, and D. M. Hatch, J. Appl. Crystallogr. 39, 607 (2006).

[29] J. B. Goodenough, Magnetism and the Chemical Bond (Interscience, New York, 1963).

[30] N. Matsubara, F. Damay, B. Vertruyen, N. Barrier, O. I. Lebedev, P. Boullay, E. Elkaïm, P. Manuel, D. D. Khalyavin, and C. Martin, Inorg. Chem. 56, 9742 (2017).

[31] J. Rodriguez-Carvajal, M. Hennion, F. Moussa, A. H. Moudden, L. Pinsard, and A. Revcolevschi, Phys. Rev. B 57, R3189 (1998).

[32] I. D. Brown and D. Altermatt, Acta Crystallogr. B 41, 244 (1985).

[33] B. E. Warren, J. Exp. Theor. Phys. 59, 693 (1941).

[34] M. Giot, L. C. Chapon, J. Androulakis, M. A. Green, P. G. Radaelli, and A. Lappas, Phys. Rev. Lett. 99, 247211 (2007).

[35] N. Terada, Y. Tsuchiya, H. Kitazawa, T. Osakabe, N. Metoki, N. Igawa, and K. Ohoyama, Phys. Rev. B 84, 064432 (2011).

[36] J. Shamblin, S. Calder, Z. Dun, M. Lee, E. S. Choi, J. Neuefeind, H. Zhou, and M. Lang, Phys. Rev. B 94, 024413 (2016).

[37] N. Matsubara, C. Martin, B. Vertruyen, A. Maignan, F. Fauth, P. Manuel, V. Hardy, D. Khalyavin, E. Elkaïm, and F. Damay, Phys. Rev. B 100, 014409 (2019).

[38] A. Prodi, E. Gilioli, A. Gauzzi, F. Licci, M. Marezio, F. Bolzoni, Q. Huang, A. Santoro, and J. W. Lynn, Nat. Mater. 3, 48 (2004).

[39] O. K. Forslund et al., Sci. Rep. 9, 1141 (2019).

[40] P. Strobel and B. Lambert-Andron, J. Solid State Chem. 75, 90 (1988).

[41] Y. Tokura and N. Nagaosa, Science 288, 462 (2000).

[42] D. D. Khalyavin, P. Manuel, W. Yi, and A. A. Belik, Phys. Rev. B 94, 134412 (2016).

[43] P. G. Radaelli, D. E. Cox, M. Marezio, and S.-W. Cheong, Phys. Rev. B 55, 3015 (1997). 
[44] N. Matsubara, S. Petit, C. Martin, F. Fauth, E. Suard, S. Rols, and F. Damay, Phys. Rev. B 100, 220406(R) (2019).

[45] F. Damay, M. Poienar, C. Martin, A. Maignan, J. RodriguezCarvajal, G. André, and J. P. Doumerc, Phys. Rev. B 80, 094410 (2009).
[46] R. D. Johnson, D. D. Khalyavin, P. Manuel, A. Bombardi, C. Martin, L. C. Chapon, and P. G. Radaelli, Phys. Rev. B 93, 180403(R) (2016).

[47] N. J. Perks, R. D. Johnson, C. Martin, L. C. Chapon, and P. G. Radaelli, Nat. Commun. 3, 1277 (2012).

[48] M. M. Vopson, Crit. Rev. Solid State Mater. Sci. 40, 223 (2015). 